\title{
An upper bound for a Hilbert polynomial on quaternionic Kähler manifolds
}

\author{
Uwe Semmelmann \& Gregor Weingart *
}

October 25, 2018

\begin{abstract}
In this article we prove an upper bound for a Hilbert polynomial on quaternionic Kähler manifolds of positive scalar curvature. As corollaries we obtain bounds on the quaternionic volume and the degree of the associated twistor space. Moreover the article contains some details on differential equations of finite type. Part of it is used in the proof of the main theorem.
\end{abstract}

AMS Subject Classification: 53C25, 58J50

\section{Introduction}

A quaternionic Kähler manifold is a $4 n$-dimensional Riemannian manifold $(M, g)$ with holonomy contained in $\mathbf{S p}(1) \cdot \mathbf{S p}(n)$. Associated with $M$ is the twistor space $Z$ which is the total space of a $\mathbb{C} P^{1}$-fibration over $M$. If the scalar curvature of $M$ is positive, the twistor space $Z$ is a Kähler-Einstein manifold admitting a complex contact structure with values in a holomorphic line bundle $L$, in particular $L^{n+1} \cong K^{*}$ is isomorphic to the anticanonical bundle $K^{*}:=\Lambda^{2 n+1} T^{1,0} Z$ of $Z$. In this situation S. Salamon introduced the Hilbert polynomial $P(r)$ of $M$ in [Sa82], which for integers $r$ is defined as the holomorphic Euler characteristic of $L^{r}$. It is easy to show that $P(r)$ for $r \in \mathbb{N}$ is given by the index of a certain twisted Dirac operator on $M$. The Hilbert polynomial $P(r)$ contains interesting information on the quaternionic Kähler manifold $M$, e. g. the dimension of the isometry group of $M$ is given as $P(1)$ according to a result of S. Salamon [Sa82].

In SW02 we developed a method based on representation theory of $\mathbf{S p}(1) \cdot \mathbf{S p}(n)$ for determining the kernel of twisted Dirac operators in terms of minimal eigenspaces of certain natural second order differential operators. This method is used here to prove our main theorem:

${ }^{*}$ The first author is a member of the European Differential Geometry Endeavour (EDGE), Research Training Network HPRN-CT-2000-00101, supported by The European Human Potential Programme. The second author is partially supported by the SFB 611 "Singuläre Phänomene und Skalierung in mathematischen Modellen" 
Theorem 1.1 Let $\left(M^{4 n}, g\right)$ be a quaternionic Kähler manifold of positive scalar curvature. The Hilbert polynomial $P(r)$ of $M$ is bounded from above by the Hilbert polynomial $P_{\mathbb{H} P}$ of the quaternionic projective space $\mathbb{H} P^{n}$ in the sense that for all integers $r \geq 0$ :

$$
0 \leq P(r) \leq P_{\mathbb{H} P^{n}}(r)=\left(\begin{array}{c}
2 n+1+2 r \\
2 n+1
\end{array}\right) .
$$

As applications we obtain in Section 3 an upper bound for the dimension of isometry group, the degree of the twistor space and the quaternionic volume. Section 1 contains a formula for the Hilbert polynomial on the Wolf spaces together with explicit expressions in several examples. The final Section 6 is more or less independent from the rest of the article. It contains details on differential operators of finite type, which gives the background for the proof of our main theorem in Section 5 .

\section{The Hilbert polynomial}

Let $\left(M^{4 n}, g\right)$ be a quaternionic Kähler manifold, a Riemannian manifold with holonomy contained in $\mathbf{S p}(1) \cdot \mathbf{S p}(n) \subset \mathbf{S O}(4 n)$. The holonomy reduction associates a globally defined vector bundle on $M$ to every representation of $\mathbf{S p}(1) \cdot \mathbf{S p}(n)$. These representations are (sums of) tensor products of $\mathbf{S p}(1)$ - and $\mathbf{S p}(n)$-representations which factor through the projection $\mathbf{S p}(1) \times \mathbf{S p}(n) \rightarrow \mathbf{S p}(1) \cdot \mathbf{S p}(n)$. In particular the standard representations $H$ and $E$ of $\mathbf{S p}(1)$ and $\mathbf{S p}(n)$ respectively induce only locally defined vector bundles whereas $\mathrm{Sym}^{2} H$ or $H \otimes E \cong T M \otimes_{\mathbb{R}} \mathbb{C}$ are globally defined.

The twistor space $Z$ of $M$ is defined as the unit sphere bundle in $\operatorname{Sym}^{2} H \subset$ End $T M$. If $M$ is a quaternionic Kähler manifold of positive scalar curvature its twistor space $Z$ is a Kähler-Einstein manifold of complex dimension $2 n+1$ endowed with a complex contact structure $\eta: T^{1,0} Z \longrightarrow L$ with values in a holomorphic line bundle $L$. Hence $L$ is an $n+1$-th root of the anticanonical bundle $K^{*}:=\Lambda^{2 n+1} T^{1,0} Z$ :

$$
L^{n+1} \cong K^{*} \text {. }
$$

The Hilbert polynomial $P(r)$ of the polarized variety $(Z, L)$ is defined as the holomorphic Euler characteristic of the line bundle $L^{r}$

$$
P(r):=\chi\left(Z, \mathcal{O}\left(L^{r}\right)\right)=\sum_{s=0}^{2 n+1}(-1)^{s} h^{s}\left(L^{r}\right)=\left\langle e^{r c_{1}(L)} \operatorname{td} Z,[Z]\right\rangle,
$$

which can be calculated from the Todd class $\operatorname{td} Z$ of $Z$ according to the Riemann-Roch Theorem. From the latter formula we conclude that $P(r)$ is a polynomial of degree $\leq 2 n+1$ in $r$ whose leading coefficient is given by the degree $\operatorname{deg} Z:=\left\langle c_{1}(L)^{2 n+1},[Z]\right\rangle$ of the twistor space $Z$ :

$$
P(r)=\frac{\operatorname{deg}(Z)}{(2 n+1) !} r^{2 n+1}+\text { terms of lower order } .
$$

Its constant term equals $P(0)=\langle\operatorname{td} Z,[Z]\rangle=1$, because $Z$ is a compact Kähler manifold of positive scalar curvature. In addition $c_{1}(L)$ is a positive class in $H^{1,1}(Z ; \mathbb{R})$, hence $L^{r}$ is 
a negative line bundle for $r<0$ and the Kodaira Vanishing Theorem asserts $h^{s}\left(L^{r}\right)=0$ for $r<0$ and $s \leq 2 n$. Moreover Serre duality implies

$$
h^{s}\left(L^{r}\right)=h^{2 n+1-s}\left(L^{-r} \otimes K\right)=h^{2 n+1-s}\left(L^{-r-n-1}\right),
$$

thus it follows the symmetry $P(r)=-P(-r-n-1)$. These properties were proved by S. Salamon in [Sa82], where he also showed that on any quaternionic Kähler manifold of non vanishing scalar curvature the space $H^{0}(Z, \mathcal{O}(L))$ of holomorphic sections of $L$ is isomorphic to the space of infinitesimal isometries of $M$ of dimension $P(1)=\operatorname{dim} \operatorname{Iso}(M, g)$. More generally an integration along the fibres results in the formula

$$
P(r)=\left\langle\hat{A}(M) \operatorname{ch~Sym}^{n+2 r} H,[M]\right\rangle,
$$

which identifies $P(r)$ with the index of a twisted Dirac operator on $M$. This observation will be our starting point for proving the upper bound for the Hilbert polynomial in Section 3 . In order to provide a closer link between the polynomial and the geometry of $M$ we need to calculate the Chern character of $\mathrm{Sym}^{n+2 r} H$ :

Lemma 2.1 The Chern character $\mathrm{ch} \mathrm{Sym}^{n+2 r} H$ of $\mathrm{Sym}^{n+2 r} H$ can be written

$$
\operatorname{ch~Sym}^{n+2 r} H=\sum_{l \geq 0} \frac{2^{2 l+1}}{(2 l+1) !} B_{2 l+1}\left(r+\frac{n+2}{2}\right) u^{l}
$$

where $u:=p_{1}(H)$ and $B_{2 l+1}(x)$ is the $2 l+1-$ th Bernoulli polynomial.

Proof: By the splitting principle we may think of the quaternionic bundle $H$ as a sum $\ell \oplus \ell^{-1}$ of two conjugated line bundles with first Chern classes $\pm \sqrt{u}$ or $u:=p_{1}(H)=c_{1}(\ell)^{2}$. Similarly we may think of $\operatorname{Sym}^{k} H, k \geq 0$, as decomposed into $\ell^{k} \oplus \ell^{k-2} \oplus \ldots \oplus \ell^{-k}$. Using the characteristic property $B_{\mu+1}(w+1)-B_{\mu+1}(w)=(\mu+1) w^{\mu}$ of the Bernoulli polynomials together with $B_{\mu+1}(w)=(-1)^{\mu+1} B_{\mu+1}(1-w)$ the Chern character of $\mathrm{Sym}^{k} H$ becomes:

$$
\begin{aligned}
\operatorname{ch~Sym}^{k} H & =e^{k \sqrt{u}}+e^{(k-2) \sqrt{u}}+\cdots+e^{-k \sqrt{u}} \\
& =\sum_{\mu \geq 0} \frac{2^{\mu}}{\mu !}\left(\sum_{\nu=0}^{k}\left(\frac{k}{2}-\nu\right)^{\mu}\right) \sqrt{u}^{\mu} \\
& =\sum_{\mu \geq 0} \frac{2^{\mu}}{(\mu+1) !}\left(B_{\mu+1}\left(\frac{k}{2}+1\right)-B_{\mu+1}\left(-\frac{k}{2}\right)\right) \sqrt{u}^{\mu} \\
& =\sum_{l \geq 0} \frac{2^{2 l+1}}{(2 l+1) !} B_{2 l+1}\left(\frac{k}{2}+1\right) u^{l} \cdot \square
\end{aligned}
$$

Using the expansion $B_{k}(x)=x^{k}-\frac{1}{2}\left(\begin{array}{l}k \\ 1\end{array}\right) x^{k-1}+\frac{1}{6}\left(\begin{array}{c}k \\ 2\end{array}\right) x^{k-2}-\frac{1}{30}\left(\begin{array}{c}k \\ 4\end{array}\right) x^{k-4} \pm \ldots$ of the Bernoulli polynomials we can expand formula (2.2) in powers of $r$ and interpret its coefficients in terms of geometric data of $M$ e. g. the leading coefficient is proportional to the volume of $M$. In fact the integral class

$$
4 u=p_{1}\left(\operatorname{Sym}^{2} H\right)=\left(\frac{\kappa}{8 \pi n(n+2)}\right)^{2} \Omega
$$


is represented by a multiple of the Kraines form $\Omega$, whose $n$-th power $\Omega^{n}=(2 n+1)$ ! $\operatorname{vol}_{M}$ is essentially the Riemannian volume form of $M$ for the canonical quaternionic orientation. Defining the quaternionic volume of $M$ as the integer

$$
v(M):=\left\langle(4 u)^{n},[M]\right\rangle=(2 n+1) !\left(\frac{\kappa}{8 \pi n(n+2)}\right)^{2 n} \operatorname{vol}(M)
$$

and substituting the expansion of $\mathrm{ch} \mathrm{Sym}^{n+2 r} H$ into equation (2.2) we find:

$$
\begin{aligned}
P(r) & =\sum_{l=0}^{n} \frac{2}{(2 l+1) !} B_{2 l+1}\left(r+\frac{n}{2}+1\right)\left\langle\hat{A}(M)(4 u)^{l},[M]\right\rangle \\
& =\frac{2}{(2 n+1) !} B_{2 n+1}\left(r+\frac{n}{2}+1\right) v(M)+\ldots \\
& =\frac{2}{(2 n+1) !} v(M) r^{2 n+1}+\frac{n+1}{(2 n) !} v(M) r^{2 n}+\ldots
\end{aligned}
$$

In particular we obtain the well-known formula $\operatorname{deg}(Z)=2 v(M)$ (c. f. Sa99). Since $P(r)$ is integer valued for all integers $r$ it follows that $P(r)$ can be written as a linear combination of binomial coefficients, i. e. there exist integers $n_{i}$ for $i=0, \ldots, 2 n+1$ with

$$
P(r)=\sum_{i=0}^{2 n+1} n_{i}\left(\begin{array}{l}
r \\
i
\end{array}\right) .
$$

On the quaternionic projective space $\mathbb{H} P^{n}$ the bundle $L$ is the square of a globally defined holomorphic line bundle $L^{\frac{1}{2}}$. Consequently the polynomial $P(r)$ has additional zeroes for $r=-1 / 2, \ldots,-n / 2$ which lead to the explicit formula:

$$
P(r)=\left(\begin{array}{c}
2 n+1+2 r \\
2 n+1
\end{array}\right) \quad \text { for } \quad r \geq 0 .
$$

\section{Indices of Twisted Dirac Operators}

According to a result of S. Salamon mentioned in Section 2 the values $P(r)$ of the Hilbert polynomial of a quaternionic Kähler manifold $M$ are indices of twisted Dirac operators $D_{\mathrm{Sym}^{n+2 r} H}$. These twisted Dirac operators belong to a two parameter family of twisted Dirac operators of particular interest in quaternionic geometry and quite a lot is known about their indices. In [SW02] we proved a general principle for operators in this family identifying their kernels with minimal eigenspaces for certain selfadjoint second order differential operators $\Delta_{\pi}$. Applying this principle for the twisted Dirac operators $D_{\operatorname{Sym}^{n+2 r} H}$ allows us to prove the estimate on the Hilbert polynomial through a detailed study of these minimal eigenspaces in the next section.

In SW02 we defined for any representation $\pi$ of $\mathbf{S p}(1) \cdot \mathbf{S p}(n)$ a natural second order differential operator $\Delta_{\pi}$ acting on sections of the associated bundle $\pi(M)$ by

$$
\Delta_{\pi}:=\nabla^{*} \nabla+2 q(R)
$$


where $q(R)$ is a selfadjoint endomorphism of $\pi(M)$ depending linearly on $R$. In terms of a local orthonormal base $\left\{\omega_{i}\right\}$ of $\mathfrak{s p}(1) \oplus \mathfrak{s p}(n) \subset \Lambda^{2} T M$ this curvature endomorphism can be written $2 q(R)=\sum \omega_{i} \cdot R\left(\omega_{i}\right)$ where $\omega_{i}$ acts via the differential of the representation of $\operatorname{Sp}(1) \cdot \operatorname{Sp}(n)$ on $\pi$. For a parallel subbundle $\pi(M) \subset \Lambda^{\bullet} T^{*} M$ the operator $\Delta_{\pi}$ coincides with the Hodge-Laplacian on forms, since definition (3.3) is nothing else but the classical Weitzenböck formula. In particular we have $2 q(R)=$ Ric on 1 -forms. Moreover it is not difficult to show that on symmetric spaces $G / K$ the operator $\Delta_{\pi}$ is the Casimir operator of $G$. For the twisted Dirac operators in question the results of [SW02 readily imply that the index of $D_{\mathrm{Sym}^{n+2 r} H}$ is given by the dimension of the minimal eigenspace of the operator $\Delta_{\mathrm{Sym}^{2 r} H}$ acting on sections of $\mathrm{Sym}^{2 r} H$ :

Lemma 3.1 Let $\left(M^{4 n}, g\right)$ be a quaternionic Kähler manifold of positive scalar curvature $\kappa$ and let $\Delta_{\mathrm{Sym}^{2 r} H}$ be the differential operator defined above acting on sections of $\mathrm{Sym}^{2 r} H$. The spectrum of $\Delta_{\mathrm{Sym}^{2 r} H}$ is bounded below by $\lambda_{2 r}:=\frac{\kappa}{2 n(n+2)} r(n+1+r)$ and:

$$
\operatorname{ker}\left(D_{\mathrm{Sym}^{n+2 r} H}\right) \cong \operatorname{ker}\left(\Delta_{\mathrm{Sym}^{2 r} H}-\lambda_{2 r}\right) .
$$

Proof: First we recall some notations and general formulas of [SW02]. The spinor bundle of $M$ is associated to the representation $S=\oplus_{i=0}^{n} R^{l, n-l}$ with $R^{l, d}:=\operatorname{Sym}^{l} H \otimes \Lambda_{0}^{d} E$. Quaternionic Kähler manifolds $M$ not isometric to $\mathbb{H} P^{n}$ are spin if and only if the quaternionic dimension $n$ is even, nevertheless this does not cause any problems as we will only consider twisted Dirac operators acting on globally defined vector bundles. If $\pi \subset S \otimes R^{l, d}$ is any representation occurring in the decomposition of the tensor product into irreducible summands, then:

$$
\Delta_{\pi}=\left.D_{R^{l, d}}^{2}\right|_{\pi(M)}+\phi(l, d) \quad \text { with } \quad \phi(l, d):=\frac{\kappa}{8 n(n+2)}(l+d-n)(l-d+n+2) .
$$

We call the representation $R^{l, d}$ a maximal twist for $\pi$ if the number $\phi(l, d)$ is maximal among all representations $R^{\tilde{l}, \tilde{d}}$ with $\pi \subset S \otimes R^{\tilde{l}, \tilde{d}}$. Using this notion we have an identification [SW02]

$$
\operatorname{ker}\left(D_{R^{l, d}}\right) \cong \bigoplus_{\pi} \operatorname{ker}\left(\Delta_{\pi}-\phi(l, d)\right)
$$

where the sum is over all $\pi \subset S \otimes R^{l, d}$ for which $R^{l, d}$ is a maximal twist. Indeed if $R^{l, d}$ is not a maximal twist for $\pi$ and so $\pi \subset S \otimes R^{\tilde{l}, \tilde{d}}$ with $\phi(\tilde{l}, \tilde{d})>\phi(l, d)$, then

$$
\left.D_{R^{l, d}}^{2}\right|_{\pi(M)}=\left.D_{R^{\tilde{l}, \tilde{d}}}^{2}\right|_{\pi(M)}+(\phi(\tilde{l}, \tilde{d})-\phi(l, d)) .
$$

Thus $D_{R^{l, d}}^{2}$ restricted to sections of $\pi(M)$ is positive and the representation $\pi$ cannot contribute to the kernel of $D_{R^{l, d}}$. Specializing to our case $R^{l, d}=\operatorname{Sym}^{n+2 r} H$ we observe that the only representation $\pi$ with maximal twist $R^{n+2 r, 0}$ is $\pi=\operatorname{Sym}^{2 r} H$, hence the lemma follows from formula (3.4).

For the canonical quaternionic orientation of $H \otimes E$ induced by the Kraines form $\Omega$, the half spin representations are given by:

$$
S^{+}:=\bigoplus_{r \equiv n(2)} R^{r, n-r} \quad S^{-}:=\bigoplus_{r \neq n(2)} R^{r, n-r} .
$$


Using the Glebsch-Gordan formula it follows immediately that $\operatorname{Sym}^{2 r} H \subset S^{+} \otimes \mathrm{Sym}^{n+2 r} H$. Hence the index of the twisted Dirac operator $D_{\mathrm{Sym}^{n+2 r} H}$ is just the dimension of its kernel:

$$
P(r)=\operatorname{index}\left(D_{\mathrm{Sym}^{n+2 r} H}\right)=\operatorname{dim} \operatorname{ker}\left(\Delta_{\mathrm{Sym}^{2 r} H}-\lambda_{2 r}\right) .
$$

The following proposition contains an estimate which is our most important technical result, which immediately implies Theorem 1.1:

Proposition 3.2 Let $M^{4 n}$ be a quaternionic Kähler manifold of positive scalar curvature

$$
\operatorname{dim} \operatorname{ker}\left(\Delta_{\mathrm{Sym}^{2 r} H}-\lambda_{2 r}\right) \leq \operatorname{dim} \operatorname{Sym}^{2 r}(H \oplus E)=\left(\begin{array}{c}
2 n+1+2 r \\
2 n+1
\end{array}\right)
$$

The proof of this proposition will be given in Section 5. As a first application we obtain the well-known upper bound for the dimension of the isometry group of a quaternionic Kähler manifold:

Corollary 3.3 Let $\left(M^{4 n}, g\right)$ be a quaternionic Kähler manifold of positive scalar curvature:

$$
\operatorname{dim} \operatorname{Iso}(M, g) \leq \operatorname{dim} \mathbf{S p}(n+1)=(n+1)(2 n+3)
$$

In Section 2 we defined the degree of the twistor space $Z$ of a quaternionic Kähler manifold. Since it appears in the leading coefficient of the Hilbert polynomial and since the twistor space of $\mathbb{H} P^{n}$ is $\mathbb{C} P^{2 n+1}$ our estimate immediately implies $\operatorname{deg}(Z) \leq \operatorname{deg}\left(\mathbb{C} P^{2 n+1}\right)$. By the definition of degree this can be reformulated into an inequality of the corresponding Chern numbers:

Corollary 3.4 Let $Z$ be the twistor space of a quaternionic Kähler manifold of positive scalar curvature:

$$
c_{1}(Z)^{2 n+1} \leq c_{1}\left(\mathbb{C} P^{2 n+1}\right)^{2 n+1}=2^{2 n+1}(n+1)^{2 n+1} .
$$

For a compact Kähler-Einstein manifold $M$ of complex dimension $m$ and positive scalar curvature C. LeBrun and S. Salamon proved the following estimate for the top power of the first Chern class (c.f. [LS94)

$$
c_{1}(M)^{m} \leq \frac{m+1}{q} c_{1}\left(\mathbb{C} P^{m}\right)^{m}
$$

where $q$ is the largest integer dividing $c_{1}(M)$, the so called index of $M$. In case $M$ admits a complex contact structure $m=2 n+1$ is odd and the index is given by $q=n+1$, so the estimate (3.6) becomes $c_{1}(M)^{m} \leq 2 c_{1}\left(\mathbb{C} P^{m}\right)^{m}$. However the compact Kähler-Einstein manifolds with complex contact structures are precisely the twistor spaces of quaternionic Kähler manifolds of positive scalar curvature (c. f. Le95 or MS96]). In this sense our estimate improves inequality (3.6) for Kähler-Einstein manifolds with complex contact structures.

Translating the estimate on the degree of the twistor space into an estimate on the quaternionic volume we obtain an upper bound for the normalized Riemannian volume of a quaternionic Kähler manifold of positive scalar curvature with the same improvement by a factor 2 compared to the estimate given in [Sa99]: 
Corollary 3.5 Let $\left(M^{4 n}, g\right)$ be a quaternionic Kähler manifold of positive scalar curvature. The quaternionic volume of $M$ is bounded from above by the quaternionic volume of $\mathbb{H} P^{n}$ :

$$
v(M) \leq v\left(\mathbb{H} P^{n}\right)=4^{n} .
$$

\section{The Hilbert Polynomial of the Wolf Spaces}

The Wolf spaces are the quaternionic Kähler symmetric spaces of positive scalar curvature $\kappa>0$. By a classical result of Wolf (c.f. Wo65) the Wolf spaces correspond up to isometry exactly to the simple compact Lie algebras. In particular there are three families of Wolf spaces in arbitrary dimensions $4 n, n \geq 2$, namely

$$
\mathbb{H} P^{n}=\frac{\mathbf{S p}(n+1)}{\mathbf{S p}(n) \times \mathbf{S p}(1)}, \quad \mathbf{G r}_{2}\left(\mathbb{C}^{n+2}\right)=\frac{\mathbf{U}(n+2)}{\mathbf{U}(n) \times \mathbf{U}(2)}, \quad \mathbf{G r}_{4}\left(\mathbb{R}^{n+4}\right)=\frac{\mathbf{S O}(n+4)}{\mathbf{S O}(n) \times \mathbf{S O}(4)}
$$

and moreover 5 exceptional Wolf spaces

$$
\frac{\mathbf{G}_{2}}{\mathbf{S O}(4)}, \quad \frac{\mathbf{F}_{4}}{\mathbf{S p}(3) \mathbf{S p}(1)}, \quad \frac{\mathbf{E}_{6}}{\mathbf{S U}(6) \mathbf{S p}(1)}, \quad \frac{\mathbf{E}_{7}}{\mathbf{S p i n}(12) \mathbf{S p}(1)}, \quad \frac{\mathbf{E}_{8}}{\mathbf{E}_{7} \mathbf{S p}(1)}
$$

in dimensions $4 n$ with $n=2,7,10,16$ and 28 respectively. It is known that up to isometry there are only finitely many quaternionic Kähler manifolds of positive scalar curvature in each dimension and it is natural to conjecture that every quaternionic Kähler manifold $M$ of positive scalar curvature has to be a Wolf space. In fact this conjecture has been proved in quaternionic dimensions $n=2,3$ and in dimension $n=4$ under the additional assumption $b_{4}(M)=1$. In all known proofs the properties of the Hilbert polynomial played a crucial role, providing the main motivation for studying it in detail, in particular we are interested in closed formulas for the Hilbert polynomial of the Wolf spaces.

Following the general construction of the Wolf spaces given in Wo65 let $\mathfrak{g}$ be a simple Lie algebra, $\mathfrak{t}$ a maximal torus and $\lambda_{\text {Wolf }} \in \mathfrak{t}^{*}$ the highest weight of the adjoint representation in a suitable ordering of roots. Let $\langle\cdot, \cdot\rangle$ be an invariant scalar product on $\mathfrak{g}$ and $\mathfrak{g}^{*}$ respectively. The crucial point is that the scalar product with $\lambda_{\text {Wolf }}$ takes exactly five different values on the set $\Delta \subset \mathfrak{t}^{*}$ of all roots:

$$
\Delta_{\hbar}:=\left\{\mu \in \Delta \mid\left\langle\lambda_{\text {Wolf }}, \mu\right\rangle=\hbar\left\langle\lambda_{\text {Wolf }}, \lambda_{\text {Wolf }}\right\rangle\right\} \quad \hbar=-1,-\frac{1}{2}, 0, \frac{1}{2}, 1
$$

and the Lie algebra of $\mathfrak{g}$ is graded correspondingly into:

$$
\mathfrak{g}=\mathfrak{g}_{1} \oplus \mathfrak{g}_{\frac{1}{2}} \oplus \mathfrak{g}_{0} \oplus \mathfrak{g}_{-\frac{1}{2}} \oplus \mathfrak{g}_{-1} \quad \mathfrak{g}_{\hbar}:=\bigoplus_{\mu \in \Delta_{\hbar}} \mathfrak{g}_{\mu}
$$

In particular the decomposition $\mathfrak{g}=\mathfrak{k} \oplus \mathfrak{p}:=\left(\mathfrak{g}_{1} \oplus \mathfrak{g}_{0} \oplus \mathfrak{g}_{-1}\right) \oplus\left(\mathfrak{g}_{\frac{1}{2}} \oplus \mathfrak{g}_{-\frac{1}{2}}\right)$ defines a symmetric pair $(\mathfrak{g}, \mathfrak{k})$ and the symmetric space corresponding to the compact real form of this symmetric pair is the Wolf space associated to the simple Lie algebra $\mathfrak{g}$. Note that the Lie subalgebra $\mathfrak{k}$ decomposes further into $\mathfrak{k}=\mathfrak{s p}(1)_{\text {Wolf }} \oplus \mathfrak{k}^{0}$ with $\mathfrak{s p}(1)_{\text {Wolf }}:=\mathfrak{g}_{1} \oplus\left[\mathfrak{g}_{1}, \mathfrak{g}_{-1}\right] \oplus \mathfrak{g}_{-1}$ and $\mathfrak{k}^{0}:=\mathfrak{g}_{0} \ominus\left[\mathfrak{g}_{1}, \mathfrak{g}_{-1}\right]$ : 
Lemma 4.1 (Uniqueness of the Minimal Representation)

For a suitable ordering of roots the Wolf root $\lambda_{\text {Wolf }}$ is the highest weight of the adjoint representation and the set of positive roots contains $\Delta^{+} \supset \Delta_{1} \cup \Delta_{\frac{1}{2}}$. Consequently the half sum of positive roots is given by

$$
\rho=\frac{n+1}{2} \lambda_{\mathrm{Wolf}}+\rho_{\mathfrak{k} 0}
$$

where $n$ is the quaternionic dimension of the Wolf space $G / K$ or equivalently $n=\frac{1}{4} \operatorname{dim} \mathfrak{p}$. Moreover for any $r \geq 0$ the finite dimensional representation $\pi=\pi_{r \lambda_{\text {Wolf }}}$ with highest weight $r \lambda_{\text {Wolf }}$ is the unique representation $\pi$ with $\operatorname{Hom}_{K}\left(\pi, \operatorname{Sym}^{2 r} H\right) \neq\{0\}$ and

$$
\mathrm{Cas}_{\pi}=\lambda_{2 r}=\frac{\kappa}{2 n(n+2)} r(n+1+r)
$$

In fact $\operatorname{dim} \operatorname{Hom}_{K}\left(\pi_{r \lambda_{\text {Wolf }}}, \operatorname{Sym}^{2 r} H\right)=1$ and $\pi_{r \lambda_{\text {Wolf }}}$ occurs once in the sections of $\operatorname{Sym}^{2 r} H$.

Proof: We start by choosing an irrational vector $v_{\mathfrak{k}^{0}} \in\left\{\lambda_{W_{\text {olf }}}\right\}^{\perp}$ orthogonal to $\lambda_{\text {Wolf }}$ to order the roots of the subalgebra $\mathfrak{k}^{0}$. To get an ordering for the roots of $\mathfrak{g}$ we make an ansatz $v=\lambda_{\text {Wolf }}+\epsilon v_{\mathfrak{k}^{0}}$ and let $\epsilon$ tend to 0 . Then the scalar product of the roots in $\Delta_{\hbar}$ with $v$ tend to $\hbar\left\langle\lambda_{\text {Wolf }}, \lambda_{\text {Wolf }}\right\rangle$. For sufficiently small $\epsilon$ the unique root $\lambda_{\text {Wolf }} \in \Delta_{1}$ will thus be maximal and $\Delta^{+} \supset \Delta_{1} \cup \Delta_{\frac{1}{2}}$. Now every root in $\Delta_{\frac{1}{2}}$ is of the form $\mu=\frac{1}{2} \lambda_{\text {Wolf }}+\operatorname{pr}_{0}(\mu)$ with $\operatorname{pr}_{0}(\mu) \in\left\{\lambda_{\text {Wolf }}\right\}^{\perp}$ and the half sum of all positive roots is given by:

$$
\begin{aligned}
\rho & =\frac{1}{2} \sum_{\mu \in \Delta_{1}} \mu+\frac{1}{2} \sum_{\mu \in \Delta_{\frac{1}{2}}} \mu+\frac{1}{2} \sum_{\mu \in \Delta_{0}^{+}} \mu \\
& =\frac{1}{2} \lambda_{\text {Wolf }}+\frac{2 n}{4} \lambda_{\text {Wolf }}+\sum_{\mu \in \Delta_{\frac{1}{2}}} \operatorname{pr}_{0}(\mu)+\rho_{\mathfrak{k}^{0}}=\frac{n+1}{2} \lambda_{\text {Wolf }}+\rho_{\mathfrak{k}^{0}}
\end{aligned}
$$

In fact the sum $\sum_{\mu \in \Delta_{\frac{1}{2}}} \operatorname{pr}_{0}(\mu)$ must vanish, because it is invariant under the Weyl group $W_{\mathfrak{k}^{0}}$ of $\mathfrak{k}^{0}$. Before we proceed we note the following relation between the scalar curvature $\kappa$ of the Wolf space $G / K$ and the length of the Wolf root:

$$
\frac{\kappa}{8 n(n+2)}=\frac{1}{4}\left\langle\lambda_{\text {Wolf }}, \lambda_{\text {Wolf }}\right\rangle
$$

This equation is invariant under rescaling of the scalar product $\langle\cdot, \cdot\rangle$ on $\mathfrak{g}$ and it is thus sufficient to check it in the Killing normalization, where the scalar curvature is $\kappa=2 n$ and the Casimir eigenvalue of the adjoint representation with highest weight $\lambda_{\text {Wolf }}$ is:

$$
1=\text { Cas }_{a d}=\left\langle\lambda_{\text {Wolf }}, \lambda_{\text {Wolf }}+2 \rho\right\rangle=(n+2)\left\langle\lambda_{\text {Wolf }}, \lambda_{\text {Wolf }}\right\rangle
$$

With all these properties of the root system of $\mathfrak{g}$ established we are now going to show that the representation $\pi_{r \lambda_{\text {Wolf }}}$ is the unique irreducible representation with Casimir eigenvalue $\operatorname{Cas}_{\pi}=r(n+1+r)\left\langle\lambda_{\text {Wolf }}, \lambda_{\text {Wolf }}\right\rangle$ occurring in the sections of Sym ${ }^{2 r} H$. Consider an irreducible representation $\pi_{\lambda}$ of $\mathfrak{g}$ with highest weight $\lambda$ and $\operatorname{Hom}_{K}\left(\pi_{\lambda}, \operatorname{Sym}^{2 r} H\right) \neq\{0\}$. 
This assumption implies that there is an element $w \in W_{\mathfrak{g}}$ of the Weyl group of $\mathfrak{g}$ such that $\left\langle w \lambda, \lambda_{\text {Wolf }}\right\rangle \geq r\left\langle\lambda_{\text {Wolf }}, \lambda_{\text {Wolf }}\right\rangle$. However the Weyl group $W_{\mathfrak{k}^{0}}$ of $\mathfrak{k}^{0}$ fixes $\lambda_{\text {Wolf }}$ and modifying $w$ by elements of $W_{\mathfrak{k}^{0}}$ we may assume that $w \lambda$ is of the form

$$
w \lambda=\frac{\delta}{2} \lambda_{\mathrm{Wolf}}+\lambda_{\mathfrak{k} 0}
$$

with dominant $\mathfrak{k}^{0}$-weight $\lambda_{\mathfrak{k}^{0}} \geq 0$. In this case we have $\delta \geq 2 r$ and hence:

$$
\begin{aligned}
\langle\lambda, \lambda+2 \rho\rangle & \geq\langle w \lambda, w \lambda+2 \rho\rangle \\
& \geq \frac{\delta(2 n+2+\delta)}{4}\left\langle\lambda_{\text {Wolf }}, \lambda_{\text {Wolf }}\right\rangle+\left\langle\lambda_{\mathfrak{k}^{0}}, \lambda_{\mathfrak{k}^{0}}+2 \rho_{\mathfrak{k}^{0}}\right\rangle \\
& \geq r(n+1+r)\left\langle\lambda_{\text {Wolf }}, \lambda_{\text {Wolf }}\right\rangle
\end{aligned}
$$

If in addition the Casimir eigenvalue of $\pi_{\lambda}$ is $\lambda_{2 r}=r(n+1+r)\left\langle\lambda_{\text {Wolf }}, \lambda_{\text {Wolf }}\right\rangle$ we must have equality everywhere in this chain of inequalities, in particular $\delta=2 r$ and $\lambda_{\mathfrak{k}^{0}}=0$ as $\lambda_{\mathfrak{k}^{0}}$ is dominant with $\left\langle\lambda_{\mathfrak{k}^{0}}, \lambda_{\mathfrak{k}^{0}}+2 \rho_{\mathfrak{k}^{0}}\right\rangle=0$. Working out the details of this argument it is easy to verify that $\pi_{r \lambda_{\text {Wolf }}}$ occurs in the sections of $\mathrm{Sym}^{2 r} H$ with multiplicity exactly 1.

The Hilbert polynomial $P(r)$ is given as the index of the twisted Dirac operator $D_{\mathrm{Sym}^{n+2 r} H}$ and by (3.5) it is the dimension of the eigenspace of $\Delta_{\mathrm{Sym}^{2 r} H}$ for the minimal eigenvalue $\lambda_{2 r}$. On symmetric spaces $G / K$ we know that the operator $\Delta_{\pi}$ coincides with the corresponding Casimir operator. Hence Frobenius reciprocity leads to:

$$
P(r)=\operatorname{dim} \operatorname{ker}\left(\Delta_{\mathrm{Sym}^{2 r} H}-\lambda_{2 r}\right)=\bigoplus_{\substack{\pi \in \hat{G} \\ \operatorname{Cas}_{\pi}=\lambda_{2 r}}} \operatorname{Hom}_{K}\left(\pi, \operatorname{Sym}^{2 r} H\right) \otimes \operatorname{dim} \pi
$$

Combining this decomposition with Lemma 4.1 we obtain an explicit formula for the polynomial $P(r)$ on compact Wolf spaces, i. e. on all symmetric quaternionic Kähler manifolds:

Corollary 4.2 The Hilbert polynomial on a compact Wolf space is given by:

$$
P(r)=\operatorname{dim}\left(\pi_{r \lambda_{A d}}\right)=\frac{n+1+2 r}{n+1} \prod_{\lambda \in \Delta_{\frac{1}{2}}}\left(1+\frac{2 r}{4\langle\rho, \lambda\rangle}\right)
$$

We will close this section in giving some explicit examples for the Hilbert polynomial on Wolf spaces. To begin with consider the 8 -dimensional Wolf space $\mathbf{G}_{2} / \mathbf{S O}(4)$. The dimension formula for $\mathbf{G}_{2}$-representations implies:

$$
P(r)=\frac{1}{120}(r+2)(3 r+5)(2 r+3)(3 r+4)(r+1) .
$$

In particular $P$ is of degree 5 with $P(0)=1$ and $P(1)=\operatorname{dim} \mathbf{G}_{2}=14$ and zeroes in $r=-1$ and $r=-2$. Moreover the leading coefficient is $\frac{3}{20}$ leading to a quaternionic volume 9 . For the real Grassmannians $\mathbf{G r}_{4}\left(\mathbb{R}^{n+4}\right)$ we obtain similarly a polynomial

$$
P(r)=\frac{(n+2 r)(n+2 r+1)(n+2 r+2)}{n^{2}(n+1)(n+2)}\left(\begin{array}{c}
n+r \\
n-1
\end{array}\right)\left(\begin{array}{c}
n+r-1 \\
n-1
\end{array}\right) .
$$


of degree $2 n+1$ with $P(0)=1$ and $P(1)=\operatorname{dim} \mathbf{S O}(n+4)$. Moreover we see that the leading coefficient is $\frac{8}{n !(n+2) !}$ so that the quaternionic volume of the real Grassmannians is given by

$$
v\left(\mathbf{G r}_{4}\left(\mathbb{R}^{n+4}\right)\right)=\frac{4}{n+2}\left(\begin{array}{c}
2 n+1 \\
n
\end{array}\right) .
$$

Doing the same calculations for the complex Grassmannians $\mathbf{G r}_{2}\left(\mathbb{C}^{n+2}\right)$ we find again a polynomial of degree $2 n+1$ with leading coefficient $\frac{2}{n !(n+1) !}$ :

$$
P(r)=\frac{n+2 r+1}{n+1}\left(\begin{array}{c}
n+r \\
r
\end{array}\right)^{2}, \quad v\left(\mathbf{G r}_{2}\left(\mathbb{C}^{n+2}\right)\right)=\left(\begin{array}{c}
2 n+1 \\
n
\end{array}\right) .
$$

\section{Minimal Eigenspaces and Their Prolongations}

In Section 3 we have seen that the value of the Hilbert polynomial $P(r)$ is the dimension of the eigenspace $\mathcal{E}=\operatorname{ker}\left(\Delta_{\mathrm{Sym}^{2 r} H}-\lambda_{2 r}\right)$ of the operator $\Delta_{\mathrm{Sym}^{2 r} H}$ acting on section of Sym $^{2 r} H$, where the constant $\lambda_{2 r}$ is the lower bound for its spectrum. In this sense $\mathcal{E}$ is the minimal eigenspace of $\Delta_{\mathrm{Sym}^{2 r} H}$. In this section we show that $\mathcal{E}$ is the kernel of a first order twistor operator on $\mathrm{Sym}^{2 r} H$ which turns out to be a differential operator of finite type. The general theory of differential equations of finite type as explained in Section 6 provides an upper bound on the dimension of $\mathcal{E}$ by the dimension of the associated total prolongation. A more direct approach to estimate the dimension of $\mathcal{E}$ taking in this section is to define a filtration on $\mathcal{E}$ such that the successive filtration quotients embed into the higher prolongations. Either way depends on knowing the prolongations explicitly and so we will determine them at the end of this section thus proving the upper bound on the dimension of $\mathcal{E}$ given in Proposition 3.2.

Recall that every representation $\pi$ of $\mathbf{S p}(1) \cdot \mathbf{S p}(n)$ gives rise to a globally defined vector bundle $\pi(M)$ on every quaternionic Kähler manifold $M$. By chance however the representations of $\mathbf{S p}(1) \cdot \mathbf{S p}(n)$ are exactly the real representations of $\mathbf{S p}(1) \times \mathbf{S p}(n)$ and are thus already defined over $\mathbb{R}$. Calculus on $M$ can thus be formulated either in terms of real or in terms of complex vector bundles only. The latter choice is more convenient as the representations $H$ and $E$ of $\mathbf{S p}(1) \times \mathbf{S p}(n)$ are both quaternionic and so we will work with complex vector bundles exclusively in particular with the complexified tangent bundle $T M \otimes_{\mathbb{R}} \mathbb{C} \cong H \otimes E$.

Consequently we will think of the covariant derivative $\nabla \psi$ of a section $\psi$ of the complex vector bundle $\operatorname{Sym}^{2 r} H$ as a section of the tensor product $\left(T^{*} M \otimes_{\mathbb{R}} \mathbb{C}\right) \otimes \operatorname{Sym}^{2 r} H$ which is isomorphic to $(H \otimes E) \otimes \operatorname{Sym}^{2 r} H \cong\left(\operatorname{Sym}^{2 r+1} H \otimes E\right) \oplus\left(\operatorname{Sym}^{2 r-1} H \otimes E\right)$. Projecting $\nabla \psi$ to both of these summands in turn defines two natural twistor operators:

$$
D_{d}^{+}: \quad \Gamma\left(\mathrm{Sym}^{2 r} H\right) \longrightarrow \Gamma\left(\mathrm{Sym}^{2 r-1} H \otimes E\right) \quad D_{u}^{+}: \quad \Gamma\left(\mathrm{Sym}^{2 r} H\right) \longrightarrow \Gamma\left(\mathrm{Sym}^{2 r+1} H \otimes E\right) .
$$

The Weitzenböck formulas of [KSW98] characterize the eigenspace $\mathcal{E}$ of $\Delta_{\mathrm{Sym}^{2 r} H}$ corresponding to the minimal eigenvalue $\lambda_{2 r}:=\frac{\kappa}{2 n(n+2)} r(n+1+r)$ as the kernel of the operator $D_{u}^{+}$: 
Proposition 5.1 Let $\psi$ be a section of $\mathrm{Sym}^{2 r} H$. Then $\Delta_{\mathrm{Sym}^{2 r} H} \psi=\lambda_{2 r} \psi$ if and only if:

$$
D_{u}^{+} \psi=0 .
$$

In this case $\psi$ satisfies additionally the equation:

$$
\left(D_{d}^{+}\right)^{*} D_{d}^{+} \psi=\frac{n}{n+1+r} \Delta_{\mathrm{Sym}^{2 r} H} \psi=\frac{\kappa r}{2(n+2)} \psi .
$$

Note that this proposition is a generalization of a lemma given in [Sa82], where it is shown in the case $r=1$ that the kernel of $D_{u}^{+}$is isomorphic to the space $H^{0}(Z, \mathcal{O}(L))$ and that it can be identified with the space of infinitesimal isometries on $M$. Indeed in this case it is easy to see that the operator $D_{d}^{+}: \operatorname{Sym}^{2} H \longrightarrow H \otimes E$ is a multiple of the codifferential $d^{*}: \Lambda^{2} T^{*} M \longrightarrow T^{*} M$ restricted to $\operatorname{Sym}^{2} H \subset \Lambda^{2}\left(T^{*} M \otimes_{\mathbb{R}} \mathbb{C}\right)$. Moreover for a section $\psi$ in the kernel of $D_{u}^{+}$we have:

$$
\Delta\left(D_{d}^{+} \psi\right)=D_{d}^{+}\left(\Delta_{\mathrm{Sym}^{2} H} \psi\right)=\frac{\kappa}{2 n} D_{d}^{+} \psi=2 \operatorname{Ric}\left(D_{d}^{+} \psi\right) .
$$

Hence for a minimal eigensection $\psi$ the coclosed 1 -form $D_{d}^{+} \psi$ is dual to a Killing vector field and vice versa.

Choose a point $x \in M$ and define the subspace $\mathcal{E}_{x}^{l}$ of the minimal eigenspace $\mathcal{E}$ by

$$
\mathcal{E}_{x}^{l}:=\left\{\psi \in \mathcal{E} \mid 0=\psi(x)=(\nabla \psi)(x)=\ldots=\left(\nabla^{l} \psi\right)(x)=0\right\} .
$$

Evidently the sequence $\mathcal{E}_{x}^{l}, l \geq 0$, of subspaces defines a descending filtration of $\mathcal{E}$

$$
\mathcal{E}:=\mathcal{E}_{x}^{-1} \supset \mathcal{E}_{x}^{0} \supset \mathcal{E}_{x}^{1} \supset \ldots \supset \mathcal{E}_{x}^{\infty}:=\bigcap_{l \geq 0} \mathcal{E}_{x}^{l}
$$

depending on the point $x \in M$. The intersection $\mathcal{E}_{x}^{\infty}$ is the space of minimal eigensections of $\Delta_{\mathrm{Sym}^{2 r} H}$ vanishing in $x$ to infinite order. However the operator $\Delta_{\mathrm{Sym}^{2 r} H}$ satisfies the strong unique continuation property (c. f. [Ka88]) and so there is no point on $M$ in which a non-trivial solution $\psi \in \mathcal{E}$ can possibly vanish to infinite order. Now $\mathcal{E}$ is the eigenspace of an elliptic differential operator on a compact manifold $M$ and is thus finite dimensional. Consequently

$$
\operatorname{dim} \mathcal{E}=\operatorname{dim}\left(\mathcal{E}_{x}^{-1} / \mathcal{E}_{x}^{0}\right)+\sum_{l \geq 0} \operatorname{dim}\left(\mathcal{E}_{x}^{l} / \mathcal{E}_{x}^{l+1}\right) .
$$

and we will use this equality to prove the upper bound on the dimension of $\mathcal{E}$ by estimating the dimension of the successive filtration quotients $\mathcal{E}_{x}^{l} / \mathcal{E}_{x}^{l+1}$. In the course of these calculations we will prove that the differential equation $D_{u}^{+} \psi=0$ is of finite type, i. e. that all its higher prolongations vanish everywhere on $M$. Indeed we will show that the $l$-th prolongation $\mathcal{A}^{(l)}$ is given as $\mathcal{A}^{(l)}=\operatorname{Sym}^{2 r-l-1} H \otimes \operatorname{Sym}^{l+1} E$ and thus vanishes for $l \geq 2 r$. For partial differential equations of finite type the assertion $\mathcal{E}_{x}^{\infty}=\{0\}$ for all $x \in M$ is a corollary of the construction of a connection on a suitable vector bundle making all solutions of the original partial differential equation parallel. For the convenience of the reader the general construction of such a connection is sketched in Section 6 . 
By definition the filtration quotient $\mathcal{E}_{x}^{-1} / \mathcal{E}_{x}^{0}$ embeds into $\operatorname{Sym}^{2 r} H$ via $[\psi] \longmapsto \psi(x)$ and hence $\operatorname{dim} \mathcal{E}_{x}^{-1} / \mathcal{E}_{x}^{0} \leq \operatorname{dim} \operatorname{Sym}^{2 r} H$. The dimension of the higher filtration quotients $\mathcal{E}_{x}^{l} / \mathcal{E}_{x}^{l+1}$ can be estimated similarly by embedding them into the higher prolongations $\mathcal{A}_{x}^{(l)}, l \geq 0$, of the symbol $\mathcal{A}^{(0)}$ of the partial differential equation $D_{u}^{+} \psi=0$ defined below. The principal symbol $\sigma_{D_{u}^{+}}:(H \otimes E) \otimes \operatorname{Sym}^{2 r} H \longrightarrow \operatorname{Sym}^{2 r+1} H \otimes E$ of the twistor operator $D_{u}^{+}$is $\mathbf{S p}(1)$. $\operatorname{Sp}(n)$-equivariant and thus fixed up to a non-vanishing constant. Redefining $D_{u}^{+}$if necessary we may assume that $\sigma_{D_{u}^{+}}$is given by $\sigma_{D_{u}^{+}}(h \otimes e \otimes \psi)=(h \cdot \psi) \otimes e$ for all $h \in H, e \in E$ and $\psi \in \operatorname{Sym}^{2 r} H$. In terms of the principal symbol we can write the partial differential equation:

$$
D_{u}^{+} \psi=\sigma_{D_{u}^{+}}(\nabla \psi)=0 .
$$

The general theory introduced in more detail in Section 0 proceeds by defining

$$
\mathcal{A}:=\left(\operatorname{Sym}^{2 r} H\right) \oplus\left(\operatorname{Sym}^{2 r-1} H \otimes E\right) \subset \operatorname{Sym}^{\leq 1}(H \otimes E) \otimes \operatorname{Sym}^{2 r} H
$$

and the symbol $\mathcal{A}^{(0)}:=\operatorname{Sym}^{2 r-1} H \otimes E$ of the differential equation $D_{u}^{+} \psi=0$ as the kernel of the principal symbol $\sigma_{D_{u}^{+}}$of $D_{u}^{+}$. This unfortunate clash of nomenclature is confusing at first but unavoidable, hopefully the reader unacquainted with these concepts will get at least an idea of why different people decided to call a subspace and an endomorphism a symbol. In order to define the higher prolongations $\mathcal{A}^{(l)}, l \geq 0$, of $\mathcal{A}^{(0)}$ let us introduce the diagonal map

$$
\Delta: \operatorname{Sym}^{k+l} T^{*} M \longrightarrow \operatorname{Sym}^{k} T^{*} M \otimes \operatorname{Sym}^{l} T^{*} M
$$

for every $k, l \geq 0$ characterized by $\Delta\left(\frac{1}{(k+l) !} \xi^{k+l}\right)=\frac{1}{k !} \xi^{k} \otimes \frac{1}{l !} \xi^{l}$ for every $\xi \in T^{*} M$. The diagonal map is coassociative in the sense that both ways to map $\operatorname{Sym}^{k+l+m} T^{*} M$ to the tensor product $\operatorname{Sym}^{k} T^{*} M \otimes \operatorname{Sym}^{l} T^{*} M \otimes \operatorname{Sym}^{m} T^{*} M$ using the diagonal $\Delta$ result in the same linear map sending $\frac{1}{(k+l+m) !} \xi^{k+l+m}$ to $\frac{1}{k !} \xi^{k} \otimes \frac{1}{l !} \xi^{l} \otimes \frac{1}{m !} \xi^{m}$. In the following it will be convenient to consider $\operatorname{Sym}^{k} T^{*} M$ as an abstract vector bundle together with a canonical embedding into the tensor product bundle $\bigotimes^{k} T^{*} M$ obtained by iterating the diagonal map as often as possible:

$$
\iota: \operatorname{Sym}^{k} T^{*} M \longrightarrow \otimes^{k} T^{*} M, \quad \frac{1}{k !} \xi^{k} \longmapsto \xi \otimes \ldots \otimes \xi .
$$

It is straightforward to check the relation $(\iota \otimes \iota) \circ \Delta=\iota$ either directly or using the definition of $\iota$ in terms of the diagonal $\Delta$ and coassociativity. With the help of the diagonal map $\Delta$ the higher prolongations $\mathcal{A}^{(l)}, l>0$, are defined as the kernels of the compositions:

$$
\begin{aligned}
\operatorname{Sym}^{l+1}(H \otimes E) \otimes \operatorname{Sym}^{2 r} H & \stackrel{\Delta}{\longrightarrow} \operatorname{Sym}^{l}(H \otimes E) \otimes(H \otimes E) \otimes \operatorname{Sym}^{2 r} H \\
\stackrel{\mathrm{id} \otimes \sigma}{\longrightarrow} & \operatorname{Sym}^{l}(H \otimes E) \otimes\left(\operatorname{Sym}^{2 r+1} H \otimes E\right)
\end{aligned}
$$

In accordance with the general interpretation of the $l$-th prolongation we have the lemma:

Lemma 5.2 Let $\psi$ be a minimal eigensection in $\mathcal{E}_{x}^{l}$, then $\left(\nabla^{l+1} \psi\right)(x) \in \mathcal{A}_{x}^{(l)}$.

Proof: It is a general fact that the iterated covariant derivative $\left(\nabla^{l+1} \psi\right)(x)$ of a section $\psi$ of a vector bundle vanishing to order $l$ in a point $x \in M$ is symmetric in all its arguments. 
For $l=1$ this amounts to say that $\left(\nabla_{X, Y}^{2} \psi\right)(x)-\left(\nabla_{Y, X}^{2} \psi\right)(x)=\left(R_{X, Y} \psi\right)(x)=0$ and the general case $l>1$ is verified using essentially the same argument with $\nabla^{l-1} \psi$ instead of $\psi$. In our case it follows that for a section $\psi \in \mathcal{E}_{x}^{l}$ vanishing in $x$ to order $l$ we we have

$$
\left(\nabla^{l+1} \psi\right)(x) \in \operatorname{Sym}^{l+1}\left(H_{x} \otimes E_{x}\right) \otimes \operatorname{Sym}^{2 r} H_{x} \subset \otimes^{l+1}\left(H_{x} \otimes E_{x}\right) \otimes \operatorname{Sym}^{2 r} H_{x}
$$

and it remains to prove that $\left(\nabla^{l+1} \psi\right)(x)$ is in the $l$-th prolongation $\mathcal{A}^{(l)}$ or equivalently is mapped to 0 under (id $\left.\otimes \sigma_{D_{u}^{+}}\right) \circ \Delta$. The crucial observation is that the diagram

$$
\begin{aligned}
& \operatorname{Sym}^{l+1}\left(H_{x} \otimes E_{x}\right) \otimes \operatorname{Sym}^{2 r} H_{x} \quad \stackrel{\iota \otimes i \mathrm{id}}{\longrightarrow} \quad \otimes^{l+1}\left(H_{x} \otimes E_{x}\right) \otimes \operatorname{Sym}^{2 r} H_{x} \\
& \Delta \downarrow \text { id } \downarrow \\
& \operatorname{Sym}^{l}\left(H_{x} \otimes E_{x}\right) \otimes\left(H_{x} \otimes E_{x}\right) \otimes \operatorname{Sym}^{2 r} H_{x} \stackrel{\iota \otimes \iota \otimes \mathrm{id}}{\longrightarrow} \otimes^{l}\left(H_{x} \otimes E_{x}\right) \otimes\left(H_{x} \otimes E_{x}\right) \otimes \operatorname{Sym}^{2 r} H_{x} \\
& \operatorname{id} \otimes \sigma_{D_{u}^{+}} \downarrow \quad \text { id } \otimes \sigma_{D_{u}^{+}} \downarrow \\
& \operatorname{Sym}^{l}\left(H_{x} \otimes E_{x}\right) \otimes\left(\operatorname{Sym}^{2 r+1} H_{x} \otimes E_{x}\right) \quad \stackrel{\iota \otimes i d}{\longrightarrow} \otimes^{l}\left(H_{x} \otimes E_{x}\right) \otimes\left(\operatorname{Sym}^{2 r+1} H_{x} \otimes E_{x}\right)
\end{aligned}
$$

commutes as a consequence of the relation $(\iota \otimes \iota) \circ \Delta=\iota$ mentioned above. Now the principal symbol $\sigma_{D_{u}^{+}}$of the twistor operator $D_{u}^{+}$is $\mathbf{S p}(1) \cdot \mathbf{S p}(n)$-equivariant and so the induced bundle homomorphism is parallel. Consequently the iterated covariant derivative $\left(\nabla^{l+1} \psi\right)(x) \in \bigotimes^{l+1}\left(H_{x} \otimes E_{x}\right) \otimes \operatorname{Sym}^{2 r} H_{x}$ of a section $\psi \in \Gamma\left(\mathrm{Sym}^{2 r} H\right)$ is mapped to

$$
\left(\text { id } \otimes \sigma_{D_{u}^{+}}\right)\left(\nabla^{l+1} \psi\right)(x)=\left(\text { id } \otimes \sigma_{D_{u}^{+}}\right)\left(\nabla^{l} \nabla \psi\right)(x)=\nabla^{l}\left(D_{u}^{+} \psi\right)(x)
$$

under the composition in the right column. In particular if $\psi \in \mathcal{E}_{x}^{l}$ is a section in the minimal eigenspace and vanishes in $x$ to order $l$ then its iterated covariant derivative $\left(\nabla^{l+1} \psi\right)(x)$ is not only in the image of $\operatorname{Sym}^{l+1}\left(H_{x} \otimes E_{x}\right) \otimes \operatorname{Sym}^{2 r} H_{x}$, but it is mapped to 0 under the composition in the right column as well. Because all horizontal arrows are injective $\left(\nabla^{l+1} \psi\right)(x)$ must be in the image of the kernel $\mathcal{A}^{(l)}$ of the composition in the left column.

Using this lemma we have an embedding of the successive filtration quotients $\mathcal{E}_{x}^{l} / \mathcal{E}_{x}^{l+1}$ into the higher prolongations $\mathcal{A}_{x}^{(l)}$ given by the well-defined map:

$$
\mathcal{E}_{x}^{l} / \mathcal{E}_{x}^{l+1} \longrightarrow \mathcal{A}_{x}^{(l)}, \quad[\psi] \longmapsto\left(\nabla^{l+1} \psi\right)(x) .
$$

Note that this map is injective by construction and hence $\operatorname{dim}\left(\mathcal{E}_{x}^{l} / \mathcal{E}_{x}^{l+1}\right) \leq \operatorname{dim} \mathcal{A}_{x}^{(l)}$. In the following lemma we will determine the higher prolongations and prove the isomorphism $\mathcal{A}^{(l)} \cong \mathrm{Sym}^{2 r-l-1} H \otimes \mathrm{Sym}^{l+1} E$, which eventually completes the proof of Proposition 3.2 by:

$\operatorname{dim} \mathcal{E} \leq \operatorname{dim} \operatorname{Sym}^{2 r} H+\sum_{l \geq 0} \operatorname{dim} \operatorname{Sym}^{2 r-l-1} H \otimes \operatorname{Sym}^{l+1} E=\operatorname{dim} \operatorname{Sym}^{2 r}(H \oplus E)$.

Lemma 5.3 The higher prolongations $\mathcal{A}^{(l)}, l \geq 0$, of $\mathcal{A}^{(0)}=\mathrm{Sym}^{2 r-1} H \otimes E$ are given by:

$$
\mathcal{A}^{(l)} \cong \operatorname{Sym}^{2 r-l-1} H \otimes \operatorname{Sym}^{l+1} E .
$$


In general knowing all prolongations $\mathcal{A}^{(l)}, l \geq 0$, is not quite sufficient as we need to know the inclusion maps $\Delta: \mathcal{A}^{(l+1)} \longrightarrow(H \otimes E) \otimes \mathcal{A}^{(l)}$ as well. In this case however these inclusion maps are $\mathbf{S p}(1) \cdot \mathbf{S p}(n)$-equivariant and thus essentially fixed by the representations $\mathcal{A}^{(l)}$.

Proof: The recursive definition of the higher prolongations discussed in more detail in Section 6 lends itself naturally to a proof by induction. We will do so by observing that both cases $l=0$ and $l=-1$ are trivial, if we interpret $\mathcal{A}^{(-1)}$ as the space $\mathrm{Sym}^{2 r} H$. According to the general theory described in Section 6 there are exact sequences characterizing $\mathcal{A}^{(l+1)}$ for all $l \geq 0$ as a subspace of $(H \otimes E) \otimes \mathcal{A}^{(l)}$

$$
0 \longrightarrow \mathcal{A}^{(l+1)} \stackrel{\Delta}{\longrightarrow}(H \otimes E) \otimes \mathcal{A}^{(l) \stackrel{\mathrm{id} \wedge \Delta}{\longrightarrow}} \Lambda^{2}(H \otimes E) \otimes \mathcal{A}^{(l-1)} .
$$

By induction hypothesis $\mathcal{A}^{(l+1)}$ is the kernel of an $\operatorname{Sp}(1) \cdot \mathbf{S p}(n)$-equivariant map

$$
(H \otimes E) \otimes\left(\mathrm{Sym}^{2 r-l-1} H \otimes \mathrm{Sym}^{l+1} E\right) \stackrel{\mathrm{id} \wedge \Delta}{\longrightarrow} \Lambda^{2}(H \otimes E) \otimes\left(\mathrm{Sym}^{2 r-l} H \otimes \mathrm{Sym}^{l} E\right)
$$

and we need to know the restriction of the diagonal map $\Delta$ to the subspace $\operatorname{Sym}^{2 r-l-1} H \otimes$ $\operatorname{Sym}^{l+1} E \subset \operatorname{Sym}^{l+1}(H \otimes E) \otimes \operatorname{Sym}^{2 r} H$ to make good use of this description. However the restriction is injective and hence fixed up to a non-vanishing constant by $\mathbf{S p}(1) \cdot \mathbf{S p}(n)-$ equivariance alone. As we are only interested in the kernel of id $\wedge \Delta$ we can ignore the constant and assume that the restriction of $\Delta$ agrees with

$$
\iota: \operatorname{Sym}^{2 r-l-1} H \otimes \operatorname{Sym}^{l+1} E \longrightarrow(H \otimes E) \otimes\left(\operatorname{Sym}^{2 r-l} H \otimes \operatorname{Sym}^{l} E\right)
$$

given by $\iota\left(\psi \otimes \frac{1}{(l+1) !} e^{l+1}\right):=\sum_{\alpha}\left(d h_{\alpha}^{b} \otimes e\right) \otimes\left(h_{\alpha} \psi \otimes \frac{1}{l !} e^{l}\right)$ for all $\psi \in \operatorname{Sym}^{2 r-l-1} H$ and $e \in E$ where $\left\{h_{\alpha}\right\}$ and $\left\{d h_{\alpha}\right\}$ is a dual pair of bases for $H$ and $H^{*}$ and $b$ is the musical isomorphism $H^{*} \longrightarrow H$ induced by the symplectic form $\sigma$ on $H$. Moreover the isomorphism

$\Lambda^{2}(H \otimes E) \stackrel{\cong}{\longrightarrow}\left(\operatorname{Sym}^{2} H \otimes \Lambda^{2} E\right) \oplus \operatorname{Sym}^{2} E,(\tilde{h} \otimes \tilde{e}) \wedge(h \otimes e) \longmapsto(\tilde{h} \cdot h \otimes \tilde{e} \wedge e) \oplus \sigma(\tilde{h}, h) \tilde{e} \cdot e$

suggests to compose id $\wedge \iota$ with the projections onto the two summands. The first composition

$$
(H \otimes E) \otimes\left(\mathrm{Sym}^{2 r-l-1} H \otimes \mathrm{Sym}^{l+1} E\right) \longrightarrow\left(\mathrm{Sym}^{2} H \otimes \Lambda^{2} E\right) \otimes\left(\mathrm{Sym}^{2 r-l} H \otimes \mathrm{Sym}^{l} E\right)
$$

sends $(\tilde{h} \otimes \tilde{e}) \otimes\left(\psi \otimes \frac{1}{(l+1) !} e^{l+1}\right)$ to $\sum_{\alpha}\left(\tilde{h} \cdot d h_{\alpha}^{b} \otimes \tilde{e} \wedge e\right) \otimes\left(h_{\alpha} \psi \otimes \frac{1}{l !} e^{l}\right)$. Hence it is the tensor product of the diagonal multiplication $H \otimes \mathrm{Sym}^{2 r-l-1} H \longrightarrow \mathrm{Sym}^{2} H \otimes \mathrm{Sym}^{2 r-l} H$ with the symplectic form in the $H$-factor with the Koszul boundary $E \otimes \operatorname{Sym}^{l+1} E \longrightarrow \Lambda^{2} E \otimes \operatorname{Sym}^{l} E$ in the $E$-factor. The diagonal multiplication with the symplectic form is always injective, whereas the kernel of the Koszul boundary is $\mathrm{Sym}^{l+2} E$ so that the kernel of the first composition is:

$$
\left(H \otimes \operatorname{Sym}^{2 r-l-1} H\right) \otimes\left(\operatorname{Sym}^{l+2} E\right) \quad \subset(H \otimes E) \otimes\left(\operatorname{Sym}^{2 r-l-1} H \otimes \mathrm{Sym}^{l+1} E\right) .
$$

Turning to the second composition

$$
(H \otimes E) \otimes\left(\operatorname{Sym}^{2 r-l-1} H \otimes \operatorname{Sym}^{l+1} E\right) \longrightarrow \operatorname{Sym}^{2} E \otimes\left(\operatorname{Sym}^{2 r-l} H \otimes \operatorname{Sym}^{l} E\right)
$$


which maps $(\tilde{h} \otimes \tilde{e}) \otimes\left(\psi \otimes \frac{1}{(l+1) !} e^{l+1}\right)$ to $-(\tilde{e} \cdot e) \otimes\left(\tilde{h} \cdot \psi \otimes \frac{1}{l !} e^{l}\right)$ we observe that it is up to sign the tensor product of the multiplication $H \otimes \mathrm{Sym}^{2 r-l-1} H \longrightarrow \mathrm{Sym}^{2 r-l} H$ with the so called Plücker differential $E \otimes \mathrm{Sym}^{l+1} E \longrightarrow \mathrm{Sym}^{2} E \otimes \mathrm{Sym}^{l} E$. The Plücker differential is injective for $l \geq 1$, whereas the kernel of the multiplication is $\operatorname{Sym}^{2 r-l-2} H$ so that for $l \geq 1$ at least the kernel of the second composition is the subspace:

$$
\left(\operatorname{Sym}^{2 r-l-2} H\right) \otimes\left(E \otimes \operatorname{Sym}^{l+1} E\right) \subset(H \otimes E) \otimes\left(\operatorname{Sym}^{2 r-l-1} H \otimes \operatorname{Sym}^{l+1} E\right) .
$$

Consequently for $l \geq 1$ the kernel of id $\wedge \iota$ must be a subspace of the intersection

$$
\left(H \otimes \operatorname{Sym}^{2 r-l-1} H\right) \otimes\left(\operatorname{Sym}^{l+2} E\right) \cap\left(\operatorname{Sym}^{2 r-l-2} H\right) \otimes\left(E \otimes \operatorname{Sym}^{l+1} E\right)
$$

of kernels of the two compositions of id $\wedge \iota$ with the projections onto the two summands $\mathrm{Sym}^{2} H \otimes \Lambda^{2} E$ and $\operatorname{Sym}^{2} E$ of $\Lambda^{2}(H \otimes E)$. Evidently this intersection is just the subspace $\mathrm{Sym}^{2 r-l-2} H \otimes \mathrm{Sym}^{l+2} E \subset(H \otimes E) \otimes\left(\mathrm{Sym}^{2 r-l-1} H \otimes \mathrm{Sym}^{l+1} E\right)$. On the other hand the kernel of id $\wedge \iota$ clearly contains $\mathrm{Sym}^{2 r-l-2} H \otimes \mathrm{Sym}^{l+2} E$ and thus agrees with it. The case $l=0$ requires extra consideration, because the kernel of the second composition is strictly larger than $\mathrm{Sym}^{2 r-2} H \otimes E \otimes E$. However this difficulty turns out to be superficial, because the intersection of the two kernels is still given by $\mathrm{Sym}^{2 r-2} H \otimes \mathrm{Sym}^{2} E$.

\section{Linear Differential Equations of Finite Type}

Among the partial differential equations the equations of finite type form a subclass with a particularly nice description of the set of solutions. It turns out that the solutions correspond to the parallel sections for a canonical connection on a suitable fibre bundle. Restricting the general case somewhat we will only consider a vector bundle $E$ over a manifold $M$ both endowed with connections and a linear partial differential equation $D(\psi)=0$ of finite type with a "parallel" linear differential operator $D$ acting on sections $\psi$ of $E$. In fact the additional assumption of $D$ being "parallel" reduces the technicalities considerably, because the principal symbol of $D$ and all its prolongations will be independent of the point of $M$ in question.

In order to make the condition of $D$ being "parallel" precise we remark that the connections on $E$ and $M$ allow us to define linear $k$-th order differential operators $\nabla^{k}, k \geq 0$, given by iterated covariant derivatives as the composition

$$
\Gamma(E) \stackrel{\nabla}{\longrightarrow} \Gamma\left(T^{*} M \otimes E\right) \stackrel{\nabla}{\longrightarrow} \Gamma\left(\otimes^{2} T^{*} M \otimes E\right) \stackrel{\nabla}{\longrightarrow} \ldots \stackrel{\nabla}{\longrightarrow} \Gamma\left(\otimes^{k} T^{*} M \otimes E\right) .
$$

It is clear that $\nabla^{k}$ encodes all $k$-th order covariant derivatives of a section and thus the operators $\nabla^{0}, \nabla^{1}, \ldots, \nabla^{k}$ together encode all partial derivatives of a given section in some trivialization of $E$. However this information is organized in a rather redundant way and for this reason we are well advised to restrict attention to the symmetrized iterated covariant derivatives, i.e. to the differential operator

$$
\text { jet }^{k}: \quad \Gamma(E) \stackrel{\nabla^{k}}{\longrightarrow} \Gamma\left(\otimes^{k} T^{*} M \otimes E\right) \stackrel{\frac{1}{k !} m}{\longrightarrow} \Gamma\left(\operatorname{Sym}^{k} T^{*} M \otimes E\right)
$$


with

$$
\operatorname{jet}_{X_{1} \ldots \cdot X_{k}}^{k} \psi:=\frac{1}{k !} \sum_{\tau} \nabla_{X_{\tau(1)}, \ldots, X_{\tau(k)}}^{k} \psi
$$

and $\mathrm{jet}^{0} \psi:=\psi$ by definition. Its symbol is the identity map from $\operatorname{Sym}^{k} T^{*} M \otimes E$ to itself and so the differential operator

$$
\mathrm{jet}^{\leq k}: \Gamma(E) \longrightarrow \Gamma\left(\operatorname{Sym}^{\leq k} T^{*} M \otimes E\right), \quad \psi \longmapsto \operatorname{jet}^{0} \psi \oplus \operatorname{jet}^{1} \psi \oplus \ldots \oplus \operatorname{jet}^{k} \psi
$$

of order $k$ from $E$ to the direct sum $\operatorname{Sym}^{\leq k} T^{*} M \otimes E:=\bigoplus_{l=0}^{k} \operatorname{Sym}^{l} T^{*} M \otimes E$ has the universal property of a jet operator. Namely for every linear differential operator $\mathfrak{D}$ of order $k$ from sections of $E$ to sections of a bundle $F$ there is a unique linear map $\sigma_{\mathfrak{D}}: \operatorname{Sym}^{\leq k} T^{*} M \otimes E \longrightarrow$ $F$ of vector bundles such that $\mathfrak{D} \psi=\sigma_{\mathfrak{D}}\left(\mathrm{jet}^{\leq k} \psi\right)$ for all sections $\psi$ of $E$. Even for naturally defined operators $\mathfrak{D}$ it is somewhat difficult to give explicit formulae for these linear maps $\sigma_{\mathfrak{D}}$ and so we need to make extensive use of the universal property in order to ensure existence of suitable linear maps below.

Consequently a general linear partial differential equation for sections $\psi$ of a vector bundle $E$ can be written $P\left(\right.$ jet $\left.{ }^{\leq k} \psi\right)=0$ for some linear map $P: \operatorname{Sym}^{\leq k} T^{*} M \otimes E \longrightarrow F$ of vector bundles and it is natural to call an equation of this form parallel if the linear map $P$ is. A differential equation which is not parallel is presumably unrelated to the affine geometry of $M$ and $E$ and it seems better not to use connections and to apply the general language of jets instead. Moreover restricting to parallel linear differential equations we avoid various technical problems, in particular the principal symbol of the differential equation is a parallel subbundle of $\operatorname{Sym}^{k} T^{*} M \otimes E$ and so all its prolongations are genuine vector bundles over $M$ with induced connections.

Let us defined the total symbol $\mathcal{A}:=\operatorname{ker} P$ as the kernel of the linear map $P$. Its principal symbol $\mathcal{A}^{(0)}:=\operatorname{ker} P \cap \mathrm{Sym}^{k} T^{*} M \otimes E$ is the kernel of the restriction res $P$ of $P$ to the subspace $\operatorname{Sym}^{k} T^{*} M \otimes E$ of polynomials of strict degree $k$. The prolongations $\mathcal{A}^{(l)}, l \geq 0$, of the symbol $\mathcal{A}^{(0)}$ are then defined as the kernels of the composition:

$$
\mathrm{Sym}^{k+l} T^{*} M \otimes E \stackrel{\Delta}{\longrightarrow} \operatorname{Sym}^{l} T^{*} M \otimes \operatorname{Sym}^{k} T^{*} M \otimes E \stackrel{\text { id } \otimes \mathrm{res}^{P}}{\longrightarrow} \operatorname{Sym}^{l} T^{*} M \otimes F,
$$

where $\Delta$ is the diagonal map as described in (5.9). For a general linear partial differential equation the $\mathcal{A}^{(l)}, l \geq 0$, are families of vector spaces over $M$ but not necessarily vector bundles, under the assumption that $P$ is parallel however $\mathcal{A}^{(l)}$ is a parallel subbundle of $\mathrm{Sym}^{k+l} T^{*} M$ and in particular is equipped with an induced connection.

According to the definition above the prolongation $\mathcal{A}^{(l)}$ is a subspace of $\operatorname{Sym}^{k+l} T^{*} M \otimes E$. On the other hand the diagonal maps $\Delta: \operatorname{Sym}^{k+l+1} T^{*} M \otimes E \longrightarrow T^{*} M \otimes \operatorname{Sym}^{k+l} T^{*} M \otimes E$ provide inclusions $\mathcal{A}^{(l+1)} \longrightarrow T^{*} M \otimes \mathcal{A}^{(l)}$ for all $l \geq 0$. These inclusions can be used to give a recursive definition of the higher prolongation $\mathcal{A}^{(l+1)}, l \geq 0$, by an exact sequence

$$
0 \longrightarrow \mathcal{A}^{(l+1)} \stackrel{\Delta}{\longrightarrow} T^{*} M \otimes \mathcal{A}^{(l) \stackrel{\mathrm{id} \wedge \Delta}{\longrightarrow}} \Lambda^{2} T^{*} M \otimes \mathcal{A}^{(l-1)},
$$

where the space $\mathcal{A}^{(-1)}$ for $l=0$ has to be interpreted as $\operatorname{Sym}^{k-1} T^{*} M \otimes E$. In fact this exact sequence can be extended to the right in the obvious way to a complex, the so called Spencer complex of the symbol $\mathcal{A}^{(0)}$, and its exactness at $T^{*} M \otimes \mathcal{A}^{(l)}$ is a direct consequence of the 
coassociativity of the diagonal map $\Delta$ combined with the fact that the Koszul complex is exact. In order to deal with all prolongations at the same time we define the total prolongation up to degree $l$ as the vector bundle

$$
\mathcal{A}^{\leq l}:=\mathcal{A} \oplus \mathcal{A}^{(1)} \oplus \ldots \oplus \mathcal{A}^{(l)},
$$

note that the first summand is $\mathcal{A}$ and not $\mathcal{A}^{(0)}$. Eventually we want to construct a sequence of injective linear maps

$$
I^{\leq l}: \mathcal{A}^{\leq l} \longrightarrow \mathrm{Sym}^{\leq k+l} T^{*} M \otimes E
$$

which has the following characteristic property:

Lemma 6.1 For every solution $\psi \in \Gamma(E)$ of the differential equation $P\left(\right.$ jet $\left.{ }^{\leq k} \psi\right)=0$ and every $l \geq 0$ there exist sections $\eta^{0} \in \Gamma(\mathcal{A})$ and $\eta^{r} \in \Gamma\left(\mathcal{A}^{(r)}\right), l \geq r \geq 1$, such that

$$
\text { jet }^{\leq k+l} \psi=I^{\leq l}\left(\eta^{0} \oplus \eta^{1} \oplus \ldots \oplus \eta^{l}\right)
$$

As the maps $I^{\leq l}$ are injective the sections $\eta^{0}, \ldots, \eta^{l}$ are uniquely determined by $\psi$.

Evidently we can simply choose the first map $I^{\leq 0}$ to be the inclusion of $\mathcal{A}=\mathcal{A}^{\leq 0}$ into $\mathrm{Sym}^{\leq k} T^{*} M \otimes E$ and $\eta^{0}:=$ jet $^{\leq k} \psi$. We postpone the proof of the lemma for general $l>0$ for a moment and give a recursive definition of the higher order maps $I \leq l, l>0$, first. For this purpose we consider the composition jet ${ }^{l} \circ P \circ$ jet $\leq k$, which is a differential operator of order $k+l$ from $E$ to $\operatorname{Sym}^{l} T^{*} M \otimes F$. The universal property of jet operators ensures the existence of a linear map

$$
P^{l}: \quad \operatorname{Sym}^{\leq k+l} T^{*} M \otimes E \longrightarrow \operatorname{Sym}^{l} T^{*} M \otimes F
$$

such that $P^{l}\left(\right.$ jet $\left.^{\leq k+l} \psi\right)=\operatorname{jet}^{l}\left(P\left(\right.\right.$ jet $\left.\left.^{\leq k} \psi\right)\right)$ for all sections $\psi$ of $E$ and symbolic calculus asserts that this map $P^{l}$ restricted to the subspace $\operatorname{Sym}^{k+l} T^{*} M \otimes E$ is simply the composition

$$
\mathrm{Sym}^{k+l} T^{*} M \otimes E \stackrel{\Delta}{\longrightarrow} \mathrm{Sym}^{l} T^{*} M \otimes \mathrm{Sym}^{k} T^{*} M \otimes E \stackrel{\mathrm{id} \otimes \operatorname{res} P}{\longrightarrow} \operatorname{Sym}^{l} T^{*} M \otimes F
$$

with kernel $\mathcal{A}^{(l)}$. The crucial step in the construction is now the choice of a partial inverse

$$
S^{l}: \operatorname{Sym}^{l} T^{*} M \otimes F \longrightarrow \operatorname{Sym}^{k+l} T^{*} M \otimes E
$$

for the restriction of $P^{l}$ to $\mathrm{Sym}^{k+l} T^{*} M \otimes E$ in the sense that $S^{l}$ maps every element in the image of $\operatorname{Sym}^{k+l} T^{*} M \otimes E$ under $P^{l}$ to some preimage, in other words $P^{l} \circ S^{l} \circ P^{l}=P^{l}$ holds true on the subspace $\mathrm{Sym}^{k+l} T^{*} M \otimes E$. Clearly such a partial inverse exists, because the kernel $\mathcal{A}^{(l)}$ of this restriction is a vector bundle on $M$ and so is its image. With the inverses $S^{l}, l \geq 0$, chosen we can inductively define the sequence the higher order maps by setting

$I^{\leq l+1}\left(\eta^{0} \oplus \ldots \oplus \eta^{l+1}\right):=I^{\leq l}\left(\eta^{0} \oplus \ldots \oplus \eta^{l}\right) \oplus\left(\eta^{l+1}-S^{l+1} P^{l+1}\left(I^{\leq l}\left(\eta^{0} \oplus \ldots \oplus \eta^{l}\right) \oplus 0\right)\right)$

for $l \geq 0$ with $I \leq 0$ being the inclusion as above. Obviously $\mathcal{A}^{(r)}, r \geq 1$ is mapped to the direct sum of the $\operatorname{Sym}^{k+l} T^{*} M \otimes E$ with $l \geq r$ only and the induced map $\mathcal{A}^{(r)} \longrightarrow \operatorname{Sym}^{k+r} T^{*} M \otimes E$ is 
simply the inclusion. Hence it follows by straightforward induction that all $I \leq l$ are injective. Moreover the maps $I^{\leq l}, l \geq 0$, have the characteristic property claimed in Lemma 6.1:

Proof: Starting the induction with $l=0$ we simply choose $\eta^{0}:=$ jet ${ }^{\leq k} \psi$ to be the $k$-jet of $\psi$ in $\mathcal{A}=\mathcal{A}^{\leq 0}$ as already discussed above. Consider now a solution $\psi \in \Gamma(E)$ and a fixed point of $M$. By induction hypothesis we may assume that in this point we have equality

$$
\text { jet }^{\leq k+l+1} \psi=I^{\leq l}\left(\eta^{0} \oplus \ldots \oplus \eta^{l}\right) \oplus \text { jet }^{k+l+1} \psi
$$

for suitably chosen $\eta^{0} \oplus \eta^{1} \oplus \ldots \oplus \eta^{l}$ in $\mathcal{A}^{\leq l}$. According to our assumption $\psi$ is a solution to $P\left(\right.$ jet $\left.^{\leq k} \psi\right)=0$ and using the fact the $P^{l+1}$ is linear we get:

$$
\begin{aligned}
P^{l+1}\left(\text { jet }^{\leq k+l+1} \psi\right) & =\operatorname{jet}^{l+1}\left(P\left(\text { jet }^{\leq k} \psi\right)\right)=0 \\
& =P^{l+1}\left(I^{\leq l}\left(\eta^{0} \oplus \ldots \oplus \eta^{l}\right) \oplus 0\right)+P^{l+1}\left(0 \oplus \text { jet }^{k+l+1} \psi\right) .
\end{aligned}
$$

In particular $P^{l+1}\left(I^{\leq l}\left(\eta^{0} \oplus \ldots \oplus \eta^{l}\right) \oplus 0\right)$ lies in the image of $\operatorname{Sym}^{k+l+1} T^{*} M \otimes E$ under $P^{l+1}$ and as $S^{l+1}$ is a partial inverse for $P^{l+1}$ on this image we may write this equality as:

$$
P^{l+1}\left(\mathrm{jet}^{k+l+1} \psi\right)+P^{l+1} S^{l+1} P^{l+1}\left(I^{\leq l}\left(\eta^{0} \oplus \ldots \oplus \eta^{l}\right) \oplus 0\right)=0 .
$$

Hence the two elements jet ${ }^{k+l+1} \psi$ and $S^{l+1} P^{l+1}\left(I^{\leq l}\left(\eta^{0} \oplus \ldots \oplus \eta^{l}\right) \oplus 0\right)$ of $\mathrm{Sym}^{k+l+1} T^{*} M \otimes E$ only differ by an element $\eta^{l+1}$ in the kernel $\mathcal{A}^{(l+1)}$ of $P^{l+1}$ restricted to $\mathrm{Sym}^{k+l+1} T^{*} M \otimes E$. In other words jet ${ }^{k+l+1} \psi=\eta^{l+1}-S^{l+1} P^{l+1}\left(I^{\leq l}\left(\eta^{0} \oplus \ldots \oplus \eta^{l}\right) \oplus 0\right)$ and we conclude

$$
\begin{aligned}
\text { jet }^{\leq k+l+1} \psi & =\text { jet }^{\leq k+l} \psi \oplus \text { jet }^{k+l+1} \psi \\
& =I^{\leq l}\left(\eta^{0} \oplus \ldots \oplus \eta^{l}\right) \oplus\left(\eta^{l+1}-S^{l+1} P^{l+1}\left(I^{\leq l}\left(\eta^{0} \oplus \ldots \oplus \eta^{l}\right) \oplus 0\right)\right) \\
& =I^{\leq l+1}\left(\eta^{0} \oplus \ldots \oplus \eta^{l} \oplus \eta^{l+1}\right)
\end{aligned}
$$

using again the induction hypothesis. It is clear that the section $\eta^{0} \oplus \ldots \oplus \eta^{l} \oplus \eta^{l+1}$ of $\mathcal{A}^{\leq l+1}$ constructed pointwise this way is a smooth section of $\mathcal{A}^{\leq l+1}$.

Definition 6.2 A partial differential equation $P\left(\right.$ jet $\left.^{\leq k} \psi\right)=0$ is called of finite type if and only if its higher prolongations $\mathcal{A}^{(l)}$ vanish everywhere on $M$ for all sufficiently large $l \gg 0$. According to the recursive definition of the prolongations $\mathcal{A}^{(l)}, l>0$, a partial differential equation is of finite type if and only if $\mathcal{A}^{(d+1)}=\{0\}$ everywhere for some $d \geq 0$.

Differential equations of finite type have a very neat characterization in terms of their symbol. Recall that a differential operator is called elliptic if and only if its principal symbol considered as a linear map is injective for every real cotangent vector. Thinking of the principal symbol as a subspace this means that the principal symbol $\mathcal{A}^{(0)}$ of an elliptic differential operator of order $k$ does not contain any $\frac{1}{k !} \xi^{k} \otimes e \neq 0$ with $\xi \in T^{*} M$ and $e \in E$ :

Theorem 6.3 A differential equation is of finite type if and only if the complexification

$$
\mathcal{A}^{(0)} \otimes_{\mathbb{R}} \mathbb{C} \subset\left(\operatorname{Sym}^{k} T^{*} M \otimes E\right) \otimes_{\mathbb{R}} \mathbb{C} \cong \operatorname{Sym}^{k}\left(T^{*} M \otimes_{\mathbb{R}} \mathbb{C}\right) \otimes\left(E \otimes_{\mathbb{R}} \mathbb{C}\right)
$$

of its principal symbol does not contain any $\frac{1}{k !} \xi^{k} \otimes e \neq 0$ with $\xi \in T^{*} M \otimes_{\mathbb{R}} \mathbb{C}$ and $e \in E \otimes_{\mathbb{R}} \mathbb{C}$. 
Using this criterion one can easily check that the operator $D_{u}^{+}$of Section 5 is of finite type. Other examples are the classical twistor operator in spin geometry or the twistor operator defining conformal Killing forms (c. f. [Se02]). Explicit upper bounds for the dimension of the solution space of a differential equation of finite type however can only be found by calculating all non-vanishing prolongations.

Of course Lemma 6.1 is valid for general parallel linear differential equations $P($ jet $\leq k \psi)=0$. What is remarkable about differential equations of finite type however is that the sequence $\mathcal{A}^{(l)}, l \geq 0$, of prolongations of the symbol becomes trivial $\mathcal{A}^{(l)}=\{0\}$ for sufficiently large $l>d \geq 0$. From this point on we have isomorphisms

$$
\mathcal{A}^{\leq d}=\mathcal{A}^{\leq d+1}=\mathcal{A}^{\leq d+2}=\ldots=\mathcal{A}^{\leq l},
$$

but we may still construct the maps $I^{\leq l}$ for all $l \geq 0$ as above. Thus for every $l \geq d$ the $k+l$-jet of some solution $\psi$ at a point of $M$ is determined by the values of the sections $\eta^{0}, \ldots, \eta^{d}$, which in turn are determined by the $k+d$-jet of $\psi$. In essence this means that from the point $l=d$ on we can calculate all higher order jets of a solution $\psi$ in a point from the $k+d$-jet of $\psi$ in this point alone. This property is very similar to the behavior of parallel sections of a vector bundle whose higher order jets at a point are all determined by the value in this point.

In order to make this analogy precise we apply the universal property of the jet operators to the differential operator $\nabla$ jet ${ }^{\leq k+d}$ of order $k+d+1$ from $E$ to $T^{*} M \otimes \operatorname{Sym}^{\leq k+d} T^{*} M \otimes E$ and find linear maps

$$
\widehat{\Delta}: \quad \operatorname{Sym}^{\leq k+d+1} T^{*} M \otimes E \longrightarrow T^{*} M \otimes \operatorname{Sym}^{\leq k+d} T^{*} M \otimes E
$$

such that $\nabla\left(\right.$ jet $\left.^{\leq k+d} \psi\right)=\widehat{\Delta}\left(\right.$ jet $\left.^{\leq k+d+1} \psi\right)$ for every section $\psi$ of $E$. Now every solution $\psi$ of the differential equation $P($ jet $\leq k \psi)=0$ corresponds to a section $\eta^{0} \oplus \ldots \oplus \eta^{d}$ of the bundle $\mathcal{A}^{\leq d}$. Recalling that $\mathcal{A}^{\leq d}$ is equipped with a natural connection we conclude

$$
\begin{aligned}
\nabla\left(I^{\leq d}\left(\eta^{0} \oplus \ldots \oplus \eta^{d}\right)\right) & =\left(\nabla I^{\leq d}\right)\left(\eta^{0} \oplus \ldots \oplus \eta^{d}\right)+\left(\mathrm{id} \otimes I^{\leq d}\right)\left(\nabla\left(\eta^{0} \oplus \ldots \oplus \eta^{d}\right)\right) \\
& =\nabla\left(\mathrm{jet}^{\leq k+d} \psi\right)=\widehat{\Delta}\left(I^{\leq d+1}\left(\eta^{0} \oplus \ldots \oplus \eta^{d}\right)\right)
\end{aligned}
$$

and so the covariant derivative of the section $\eta^{0} \oplus \ldots \oplus \eta^{d}$ is completely determined by

$$
\left(\mathrm{id} \otimes I^{\leq d}\right)\left(\nabla\left(\eta^{0} \oplus \ldots \oplus \eta^{d}\right)\right)=\widehat{\Delta}\left(I^{\leq d+1}\left(\eta^{0} \oplus \ldots \oplus \eta^{d}\right)\right)-\left(\nabla I^{\leq d}\right)\left(\eta^{0} \oplus \ldots \oplus \eta^{d}\right)
$$

as $\operatorname{id} \otimes I^{\leq d}$ is injective. We may reformulate this property in terms of the differential operator

$$
\begin{aligned}
D\left(\eta^{0} \oplus \ldots \oplus \eta^{d}\right):= & \left(\mathrm{id} \otimes I^{\leq d}\right)\left(\nabla\left(\eta^{0} \oplus \ldots \oplus \eta^{d}\right)\right) \\
& \quad-\left(\widehat{\Delta}\left(I^{\leq d+1}\left(\eta^{0} \oplus \ldots \oplus \eta^{d}\right)\right)-\left(\nabla I^{\leq d}\right)\left(\eta^{0} \oplus \ldots \oplus \eta^{d}\right)\right)
\end{aligned}
$$

from sections of $\mathcal{A}^{\leq d}$ to $T^{*} M \otimes \mathrm{Sym}^{\leq k+d} T^{*} M \otimes E$, whose principal symbol is the inclusion:

$$
\left(\mathrm{id} \otimes I^{\leq d}\right): \quad T^{*} M \otimes \mathcal{A}^{\leq d} \longrightarrow T^{*} M \otimes \mathrm{Sym}^{\leq k+d} T^{*} M \otimes E .
$$


Every section $\eta^{0} \oplus \ldots \oplus \eta^{d}$ of $\mathcal{A}^{\leq d}$ which corresponds to a solution $\psi$ of the differential equation $P\left(\right.$ jet $\left.^{\leq k} \psi\right)=0$ is killed by $D$. More precisely the solutions $\psi$ of the original differential equation correspond bijectively to the sections of $\mathcal{A}^{\leq d}$ in the kernel of the operator $D$. In fact the differential operator $D$ is essentially the restriction of the so called canonical connection

$$
\nabla^{\mathrm{can}}: \Gamma\left(\mathrm{Sym}^{\leq k+d+1} T^{*} M \otimes E\right) \longrightarrow \Gamma\left(T^{*} M \otimes \mathrm{Sym}^{\leq k+d} T^{*} M \otimes E\right)
$$

or the "naive" Spencer operator to the image of $\mathcal{A}^{\leq d}$ under $I^{\leq d+1}$, and it is well-known and easy to prove that the sections of $\mathrm{Sym}^{\leq k+d+1} T^{*} M \otimes E$ parallel under the canonical connection $\nabla^{\text {can }}$ are precisely the $k+d+1$-jets jet ${ }^{k+d+1} \psi$ of sections $\psi$ of $E$.

Now we have succeeded in identifying the space of solutions to the original differential equation $P\left(\right.$ jet $\left.^{\leq k} \psi\right)=0$ with the kernel of the differential operator $D$ and one may wonder what we have achieved at all. However the differential operator $D$ has injective symbol and thus the condition $D\left(\eta^{0} \oplus \ldots \oplus \eta^{d}\right)=0$ is stronger than $\eta^{0} \oplus \ldots \oplus \eta^{d}$ being parallel for a suitable connection on $\mathcal{A}^{\leq d}$. Indeed for a first order differential operator $\mathfrak{D}: \Gamma(\mathcal{A}) \longrightarrow \Gamma(F)$ with injective symbol $\sigma_{\mathfrak{D}}$ the image of $\sigma_{\mathfrak{D}}$ is a subbundle of $F$ and hence we can choose a smooth projection pr onto this image. The composition $\sigma_{\mathfrak{D}}^{-1} \circ \mathrm{pr} \circ \mathfrak{D}$ is a differential operator of first order from $\mathcal{A}$ to $T^{*} M \otimes \mathcal{A}$ with principal symbol given by the identity of $T^{*} M \otimes \mathcal{A}$. In other words $\sigma_{\mathfrak{D}}^{-1} \circ \operatorname{pr} \circ \mathfrak{D}$ is a connection on $\mathcal{A}$ making sections in the kernel of $\mathfrak{D}$ parallel. In our case we have to compose $D$ with some linear projection

$$
\text { pr }: \quad T^{*} M \otimes \operatorname{Sym}^{\leq k+d} T^{*} M \otimes E \longrightarrow\left(\mathrm{id} \otimes I^{\leq d}\right)\left(T^{*} M \otimes \mathcal{A}^{\leq d}\right)
$$

onto the image of $T^{*} M \otimes \mathcal{A}^{\leq d}$ under id $\otimes I^{\leq d}$ in order to obtain a connection on the vector bundle $\mathcal{A}^{\leq d}$ such that every solution to the original equation corresponds to a parallel section of $\mathcal{A}^{\leq d}$. However the nice feature of the operator $D$ of classifying the solutions completely may get lost in projecting $D$ to this Killing connection, in other words there may be sections of $\mathcal{A}^{\leq d}$ parallel for the Killing connection but not killed by $D$, which do not correspond to any solution of the original equation at all:

Proposition 6.4 Let $P\left(\right.$ jet $\left.^{\leq k} \psi\right)=0$ be a parallel linear differential equation of finite type with $\mathcal{A}^{(d+1)}=\{0\}$ on a connected manifold $M$. The dimension of the space of solutions of this differential equation is bounded above by the dimension of $\mathcal{A}^{\leq d}$. Moreover the solutions of the differential equation correspond bijectively to sections of the vector bundle $\mathcal{A} \leq d$ parallel for every connection on $\mathcal{A} \leq d$ constructed from projecting the canonical connection $D$ as above.

\section{References}

[Ka88] Kazdan, J. Unique continuation in geometry. Comm. Pure Appl. Math. 41 (1988), no. 5, 667-681.

[KSW98] Kramer, W., Semmelmann, U. and Weingart, G. Quaternionic Killing spinors. Ann. Glob. Anal. Geom. 16 (1998), 63-87.

[LS94] LeBrun, C. and Salamon, S. Strong rigidity of positive quaternion-Kähler manifolds. Invent. Math. 118 (1994), no.1, 109-132. 
[Le95] LeBrun, C. Fano manifolds, contact structures, and quaternionic geometry. Internat. J. Math. 6 (1995), no. 3, 419-437.

[MS96] Moroianu, A. and Semmelmann, U. Kählerian Killing spinors, complex contact structures and twistor spaces. C. R. Acad. Sci. Paris Ser. I Math. 323 (1996), no. 1, 57-61.

[Sa82] Salamon, S.M. Quaternionic Kähler manifolds, Invent. Math. 67 (1982), 143171.

[Sa99] Salamon, S.M. Quaternion-Kähler geometry. Surveys in differential geometry: essays on Einstein manifolds, 83-121, Surv. Differ. Geom., VI, Int. Press, Boston, MA, (1999).

[SW02] Semmelmann, U. and Weingart, G., Vanishing theorems for quaternionic Kähler manifolds , J. Reine Angew. Math. 544 (2002), 111-132.

[Se02] Semmelmann, U., Conformal Killing forms on Riemannian manifolds, preprint (2002), math.DG/0206117.

[Wo65] Wolf, J., Complex homogeneous contact manifolds and quaternionic symmetric spaces, J. Math. Mech. 14 (1965), 1033-1047; 